Revue des patrimoines

$43 \mid 2021$

Des écoles d'art académiques aux écoles d'art : des collections et des lieux, un patrimoine à valoriser

\title{
Un creuset artistique et patrimonial à redécouvrir
}

Les collections des écoles de dessin et des académies d'art du XVIII ${ }^{\mathrm{e}}$ siècle An artistic and heritage melting pot to rediscover. The collections of $18^{\text {th }}$ century drawing schools and art academies

\section{Anne Perrin Khelissa et Émilie Roffidal}

\section{(e) OpenEdition}

Journals

Édition électronique

URL : http://journals.openedition.org/insitu/28557

DOI : $10.4000 /$ insitu. 28557

ISSN : 1630-7305

Éditeur

Ministère de la Culture

Référence électronique

Anne Perrin Khelissa et Émilie Roffidal, « Un creuset artistique et patrimonial à redécouvrir », In Situ [En ligne], 43 | 2021, mis en ligne le 20 janvier 2021, consulté le 20 janvier 2021. URL : http:// journals.openedition.org/insitu/28557 ; DOI : https://doi.org/10.4000/insitu.28557

Ce document a été généré automatiquement le 20 janvier 2021.

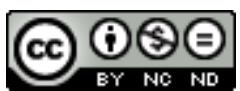

In Situ Revues des patrimoines est mis à disposition selon les termes de la licence Creative Commons Attribution - Pas d'Utilisation Commerciale - Pas de Modification 4.0 International. 


\section{Un creuset artistique et patrimonial à redécouvrir}

Les collections des écoles de dessin et des académies d'art du XVIII ${ }^{\mathrm{e}}$ siècle

An artistic and heritage melting pot to rediscover. The collections of $18^{\text {th }}$ century drawing schools and art academies

Anne Perrin Khelissa et Émilie Roffidal

1 En novembre 2017, les deuxièmes journées d'étude du programme de recherche ACA$\mathrm{RES}^{1}$, «Les académies d'art et leurs réseaux au Siècle des Lumières », portaient sur la mobilité des artistes et la dynamique des institutions. Les actes de la rencontre se concluent par cette perspective : «L'identification d'une culture de ville comme moteur diffus de la structure des académies et des écoles de dessin provinciales conduit à s'interroger sur leur action pour la protection du patrimoine. Quel rôle les académies et les écoles de dessin jouent-elles dans l'expansion d'une culture artistique locale? Quels moyens sont employés outre la construction d'un récit historique (Vies d'artistes, guides des villes) et l'organisation d'un tourisme urbain («tours de ville» organisés par les membres des académies)? $»^{2}$. Autant de questions laissées en suspens que nous souhaitons éclaircir à présent.

Dans ses travaux sur l'histoire des musées et du patrimoine en France, Dominique Poulot tient compte du type particulier des collections des écoles de dessin, en s'appuyant sur des exemples marquants: grâce au legs en 1754 d'Antoine Ferrand de Monthelon, professeur de l'école de dessin de Reims, le musée des Beaux-Arts de la ville, fondé en 1794 à partir des saisies révolutionnaires, se voit enrichi d'une série de pièces remarquables de l'école allemande, dont des détrempes de Cranach le Jeune ${ }^{3}$; à Amiens, en 1782, la municipalité ordonne la création d'un "salon » à côté de l'école de dessin pour mieux former les artistes et la population dans son entier, dispositif qui s'observe également à Toulouse et Dijon et qui préfigure les galeries publiques des musées 4 .

3 Pour ce qui concerne la bibliographie spécifique sur les écoles de dessin et les académies d'art provinciales, l'ouvrage de référence d'Agnès Lahalle ${ }^{5}$, publié en 2006, 
insiste sur la finalité pédagogique des collections, sans aller dans le détail des œuvres rassemblées, leur nature, leur provenance et leur historique. Son propos se situe plutôt au cœur des logiques de formation des écoles de dessin et du fonctionnement de leur enseignement (rapport professeur-élève, composition des cours, etc.). Compte tenu de la diversité des cas traités - une cinquantaine d'établissements sont créés entre 1740 et 1791 - l'entreprise de renouer avec l'histoire des collections est ardue et une synthèse pour l'ensemble de la France reste à écrire.

sont les prémices de ce chantier que nous proposons d'esquisser ici, en écho à l'exposition virtuelle sur ces collections spécifiques en France au XVIII ${ }^{e}$ siècle ${ }^{6}$. S'il est possible de s'appuyer sur des publications monographiques, des catalogues et des travaux universitaires solides ${ }^{7}$ ainsi que sur une actualité riche à travers les récentes expositions des musées des Beaux-Arts de Marseille et de Montpellier ${ }^{8}$, il faut encore en esquisser une vue d'ensemble.

Contrairement à l'Académie royale de peinture et de sculpture de Paris dont les sources documentaires sont localisées et plus homogènes ${ }^{9}$, la disparité de situation des villes rend l'enquête dans les archives complexe. Les processus de collecte, les contextes d'origine sont individualisés. La continuité des fonds jusqu'à nos jours est, quant à elle, aléatoire. La plupart du temps, les collections ont été dispersées sous la Révolution française pour être réattribuées diversement aux écoles centrales sous le Directoire, puis aux écoles des beaux-arts, musées locaux et bibliothèques municipales au $\mathrm{XIX}^{\mathrm{e}}$ siècle. Toutes ces entités patrimoniales devront être considérées mais, au-delà d'une démarche qui chercherait uniquement à identifier et à attribuer des œuvres encore conservées, nous voudrions poser une autre question : quels sont les enjeux de ces rassemblements singuliers d'œuvres? Ils ne ressemblent ni à des fonds d'atelier privé ni à des collections d'un particulier.

6 Pour approfondir cette réflexion, nous commencerons par évoquer l'originalité de la constitution des collections. Nous verrons ensuite qu'elles représentent un compendium d'une certaine idée de l'art, mue à cette époque par de fortes dynamiques culturelles et économiques. Enfin, il s'agira d'envisager la matérialité des œuvres, toujours à partir d'exemples choisis.

\section{Originalité de la constitution des collections}

7 Dans l'histoire des collections françaises, celles issues des écoles de dessin et des académies ont un statut à part. Elles cristallisent un ensemble de processus et de paradigmes structurant en histoire de l'art. Leur spécificité tient tout d'abord à leur mode de constitution. Elles ne sont pas le fruit de l'action unique d'un individu, ou d'une même famille, dans le cas d'une transmission d'héritage. Elles résultent d'actions collectives parfois complexes mettant en jeu des individus aux statuts et aux intérêts divers. Elles traduisent et forgent a posteriori une certaine identité artistique locale. Le résultat s'avère donc souvent composite. Quoi de commun, si ce n'est de partager des modèles artistiques, entre les collections de l'école de dessin de Saint-Quentin, établissement fondé par Maurice Quentin de La Tour en $1782^{10}$ dans une visée d'application directe aux arts "méchaniques ${ }^{11}$ ", et une collection plus généraliste comme celle de Lille ${ }^{12}$ ? Dans le premier cas, les œuvres de la main du pastelliste et d'autres maîtres qu'il rassemblait pour servir de modèles restèrent sans affectation à son décès, sans descendant. Son frère chercha à vendre ce fonds, sans succès, et décida 
donc d'en faire don à la Ville. En 1810, il servit à l'école de dessin avant de rejoindre en 1877 le siège du nouveau musée Antoine-Lécuyer ${ }^{13}$. Dans le second cas, le processus d'acquisition du fonds est plus habituel. L'album de dessins conservé aujourd'hui à la bibliothèque municipale de Lille provient de l'achat d'un professeur qui manquait de bons supports pour étayer son enseignement ${ }^{14}$.

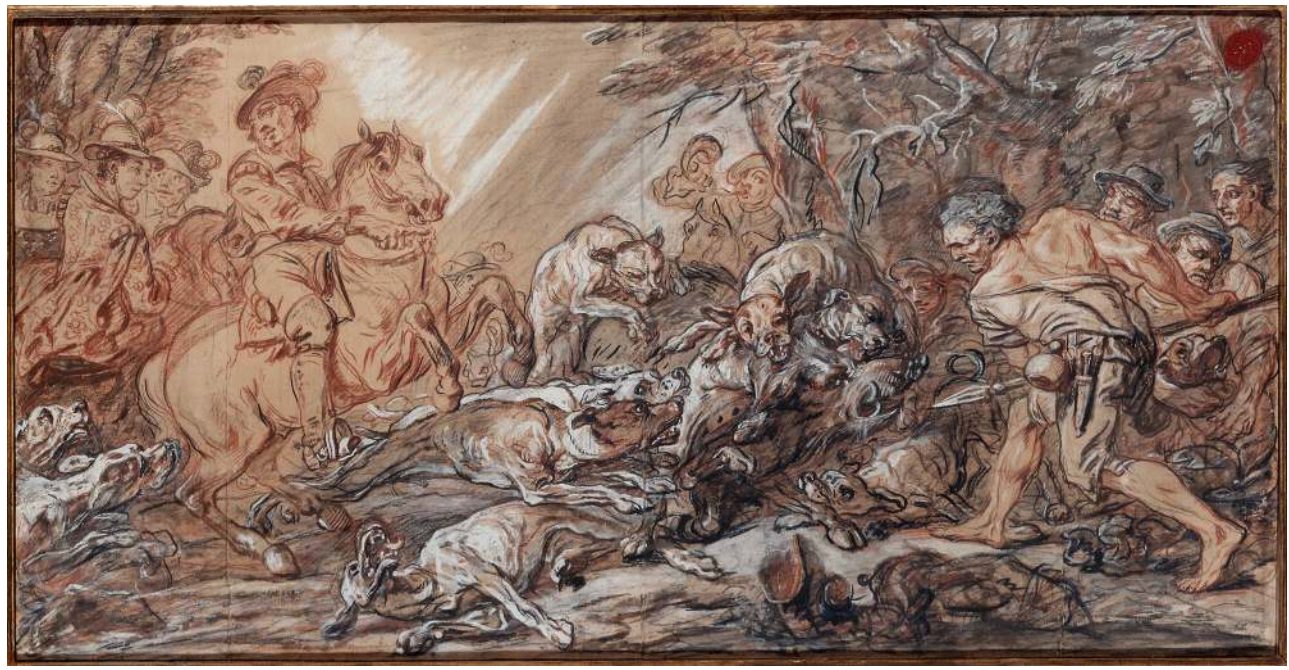

L'Europe sous l'emblème d'une chasse au sanglier, Charles Parrocel, vers 1745, sanguine, pierre noire, fusain, lavis et autres techniques sur papier, conservé au musée Antoine-Lécuyer, Saint-Quentin.

Reproduction Hervé Lewandowski (RMN-Grand Palais).

8 Par rapport à un fonds d'atelier traditionnel, la mémoire artistique composée par l'établissement pédagogique est amplifiée. La réunion d'œuvres, croquis, dessins d'exécution, estampes, peintures, modelli, sculptures, met en dialogue l'univers des artistes avec celui des collectionneurs. En effet, plusieurs curieux et amateurs locaux, soucieux d'inscrire et de pérenniser leur nom au sein d'un établissement clef dans la cité léguèrent ou donnèrent plusieurs œuvres à l'école. C'est le cas parmi d'autres de Pierre-Joseph-Laurent de Gaillard de Longjumeau qui offre en 1766, par testament à l'académie de peinture et de sculpture de Marseille, deux grands in-folio contenant plusieurs dizaines de dessins des grands maîtres européens ${ }^{15}$.

9 Pour compléter l'apport des professeurs et des donateurs privés, des achats ponctuels sont effectués par les institutions, sur fonds personnels ou sur subvention de la municipalité. Les choix portent, non pas seulement sur des modèles artistiques admis comme indispensables à une culture "classique ", mais aussi eu égard à leur utilité pratique pour les secteurs industriels, en particulier les manufactures d'objets décoratifs comme la céramique et le textile. Réunir des recueils de planches de fleurs, de fruits, d'ornements, motifs privilégiés de ce genre de produits, répond à un besoin d'efficacité formelle dans un marché du luxe et du demi-luxe concurrentiel ${ }^{16}$. À leurs contacts, les élèves s'entraînent à saisir des formes, préciser les lignes de contours et les dynamiques de composition, répéter des formules transposables sur différents supports. 


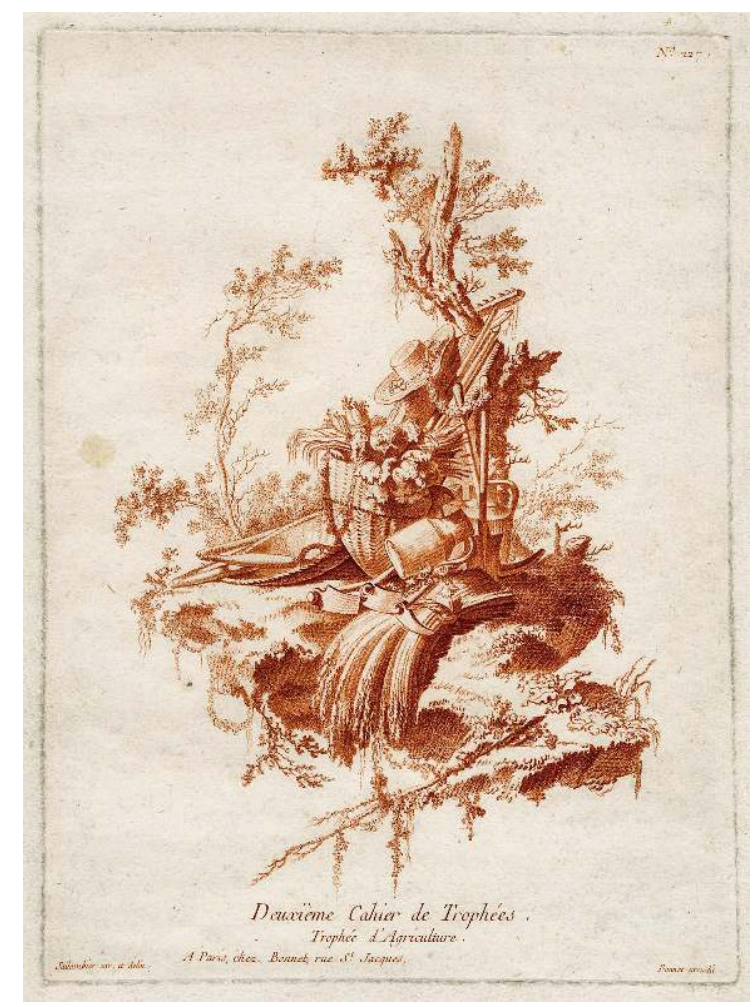

Estampe extradite du Deuxième cahier de trophées d'Henri Sallambier, vers 1775-1776, Paris, chez Bonnet. Estampe conservée au British Museum, Londres.

Reproduction The Trustees of the British Museum.

Ce souci d'application pratique et la dimension technique qu'assument plusieurs écoles de dessin dans cette seconde moitié du XviII ${ }^{e}$ siècle les rapprochent des écoles des ponts et chaussées qui émergent sur le territoire dans ces mêmes années $1740-1750^{17}$. À Bordeaux par exemple, l'Académie de peinture, sculpture et architecture civile et navale assume une formation à destination des ingénieurs et des architectes travaillant pour le port, avec à partir de 1773 quatre professeurs spécialisés dans ces domaines ${ }^{18}$. Dans les salles de l'école de dessin dirigée par Jean-Baptiste Descamps à Rouen, les élèves peuvent observer une maquette du projet de l'hôtel de ville par AntoineMatthieu Le Carpentier qui se trouve aujourd'hui dans les salles du musée des Beaux$\operatorname{Arts}^{19}$. 


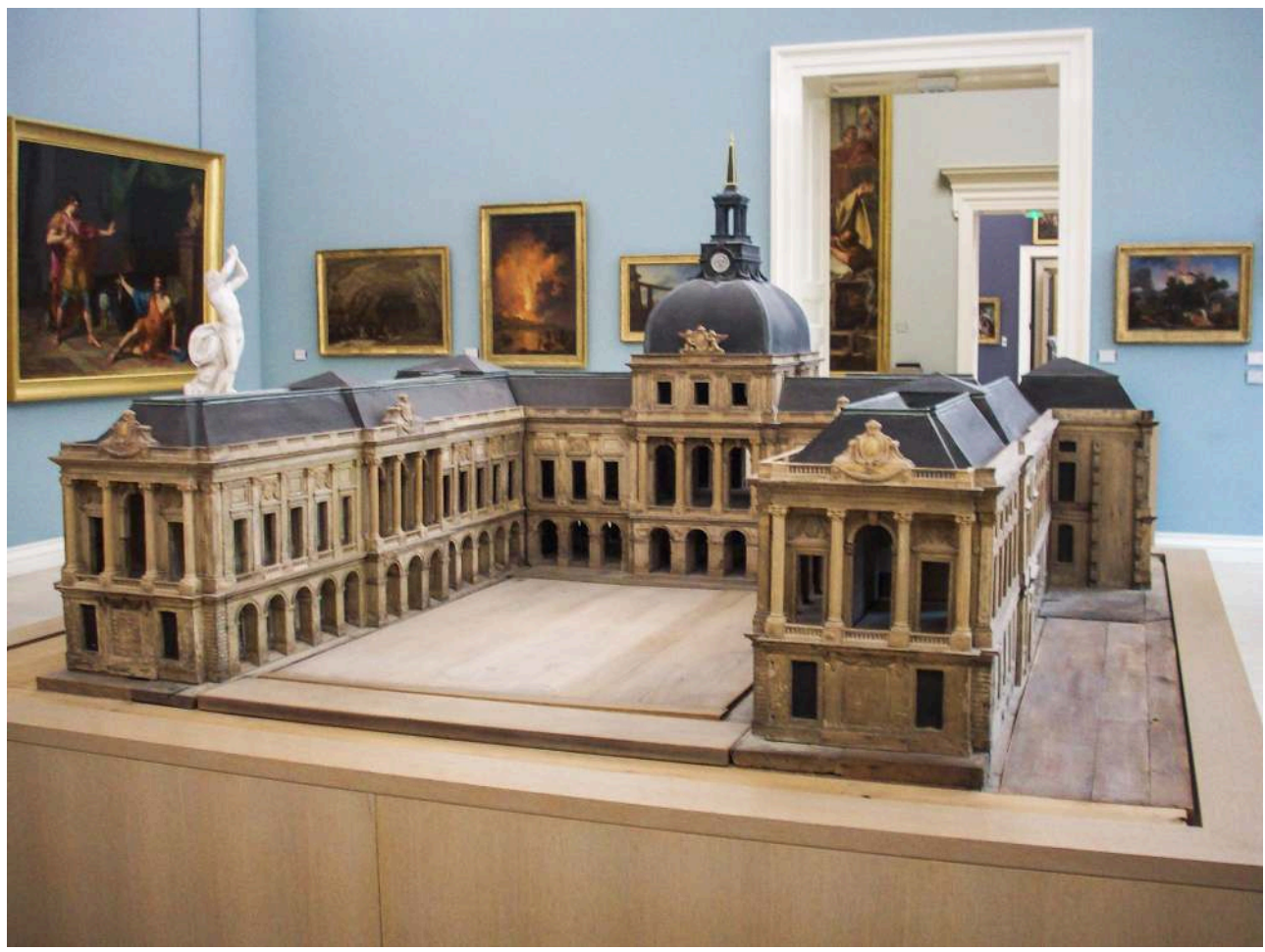

Maquette de l'hôtel de ville conçu par Antoine-Matthieu Le Carpentier, 1758, bois de chêne, conservée au Musée des beaux-arts, Rouen.

(C) Giogo (sous licence CC BY-SA).

11 La production d'œuvres réalisées spécifiquement pour les académies représente un autre mode d'enrichissement des collections : copies d'œuvres célèbres sous forme de tableaux, de dessins ou d'estampes; œuvres originales puisant dans de nouveaux répertoires; morceaux de réception et morceaux de prix voués à être conservés par les institutions. Ces témoignages, de qualité variable, mettent en lumière des noms peu connus, d'artistes professionnels et d'artistes amateurs. Ainsi, le pastel représentant un ermite lisant ${ }^{20}$ est de la main du marquis de Bonnac, François-Armand d'Usson, reçu associé honoraire en 1773 à l'Académie royale de peinture, sculpture et architecture de Toulouse avec ce morceau de réception ${ }^{21}$. Pour Bordeaux, on peut citer Le Tombeau d'Élysée, morceau de réception de Jean-Joseph Taillasson, peint à Rome et envoyé à l'académie des arts en $1774^{22}$. 


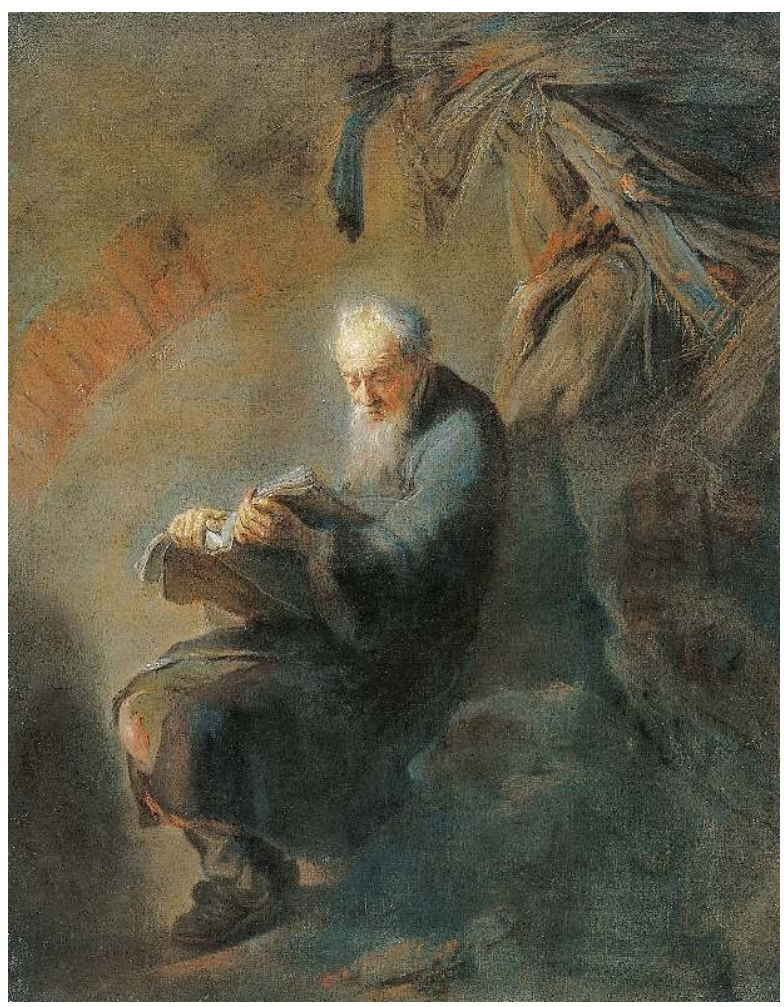

Ermite lisant, François-Armand d'Usson de Bonnac, vers 1773, pastel sur toile, conservée au Musée des Augustins, Toulouse. Conservée dans les collections de l'Académie royale de peinture, sculpture et architecture de Toulouse depuis 1773, l'œuvre entre au musée en 1795 en tant que saisie révolutionnaire.

Reproduction D. Martin (Musée des Augustins de Toulouse).

Dans le cas de Toulouse et de quelques autres villes du royaume, de telles œuvres bénéficiaient d'une réception élargie grâce à des expositions publiques organisées régulièrement par l'académie ${ }^{23}$. À Dijon, l'école de dessin fondée en 1766 est complétée en 1787 d'un muséum qui sert à exposer les ouvrages des académiciens à côté d'autres productions de maîtres reconnus ${ }^{24}$. Ce terme de "musée » renvoie à un espace d'exposition pensé également comme lieu d'apprentissage. Par les modalités de leur exposition, la possible agentivité des œuvres est clairement perceptible : elles créent une dynamique d'émulation et favorisent les commentaires de comparaison de la part du public. Une œuvre comme l'Ariane dans l'île de Naxos (1725) de Jean-François de Troy était présente dans la salle des assemblées de l'école centrale de l'Hérault en 1797, accueillant les élèves primés lors de cérémonies officielles. Elle représentait à ce titre un élément central du dispositif social, mondain, culturel de la vie artistique de l'époque ${ }^{25}$. 


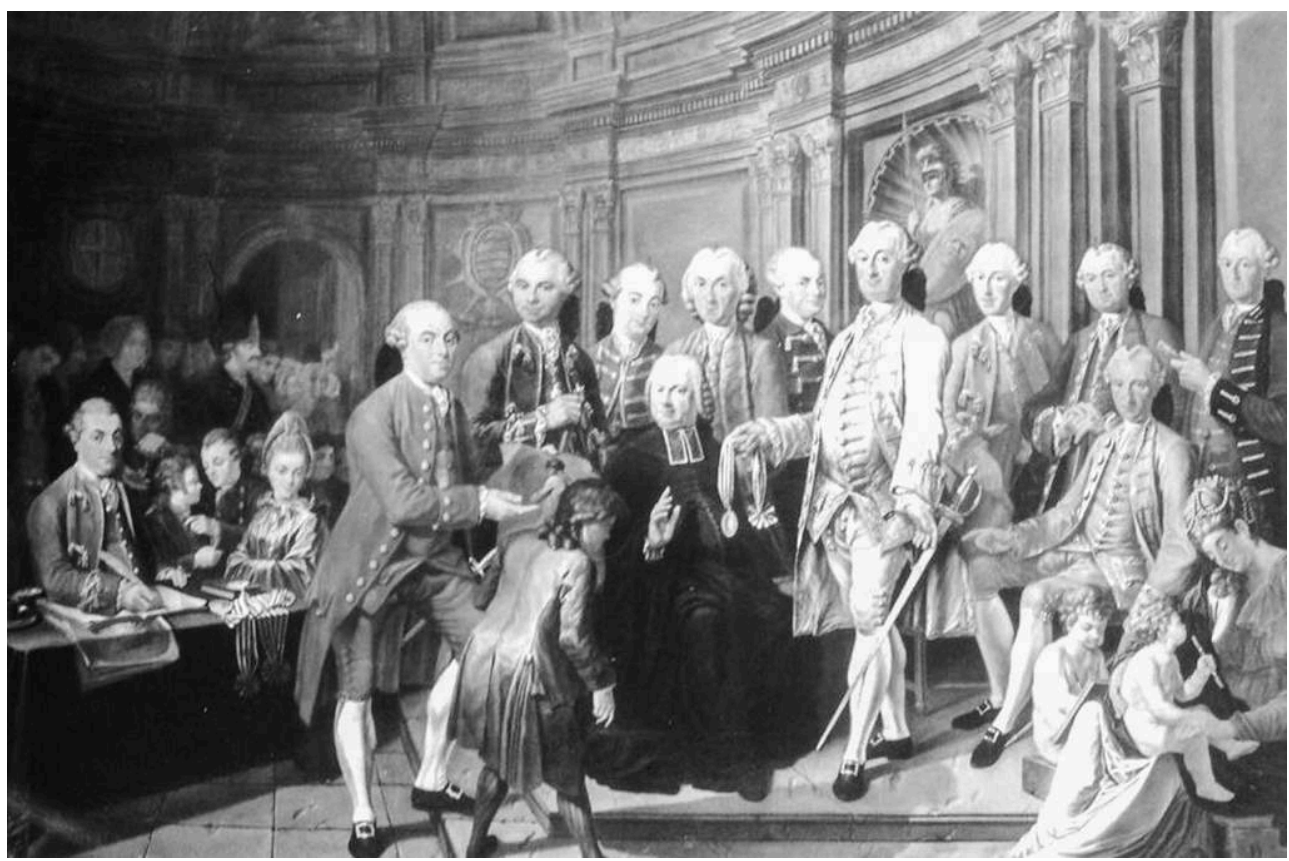

La Distribution des prix de l'Académie de dessin et de peinture de la Ville de Dunkerque, Nicolas Truit, huile sur toile, conservée au musée des Beaux-Arts de Dunkerque.

Reproduction musée des Beaux-Arts de Dunkerque.

13 Bien moins prestigieux mais cependant constitutif d'une part importante des collections, ces travaux des artistes récipiendaires et des officiers des écoles traduisent les attendus de la formation du goût de l'époque. Ils constituent aujourd'hui un patrimoine méconnu du grand public. À Valenciennes, François-Joseph Duret présente un Diogène qui lui sert ensuite pour devenir professeur de l'école en $1788^{26}$; Olivier Le May intègre l'établissement en 1785 avec Herminie chez les bergers ${ }^{27}$. À Saint-Quentin, Jérôme Preudhomme, avec La Mort de Lucrèce, remis en 1784, pose un jalon dans sa carrière avant d'accéder au poste de professeur-directeur de l'école, puis à la fonction d'officier municipal ${ }^{28}$. Il reste à établir le corpus de ces œuvres dispersées en plusieurs endroits de province, pour réfléchir notamment aux genres, aux thèmes, aux références, privilégiés par ces artistes en devenir. 


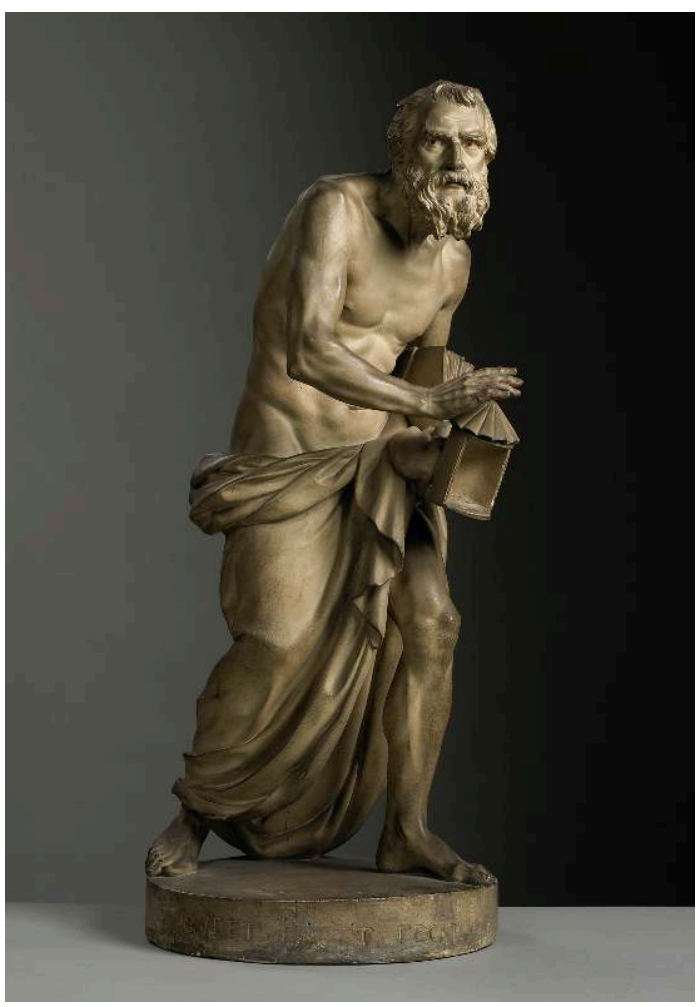

Diogène, François-Joseph Duret, 1788, plâtre original, morceau de réception conservé au musée des Beaux-Arts de Valenciennes.

(c) Photo René-Gabriel Ojéda (RMN-Grand Palais).

\section{Des collections patrimoniales à l'aune de l'histoire du goût}

Les documents d'archives disponibles pour documenter ces collections restent le plus souvent parcellaires et incomplets. L'inventaire stipulé clairement comme celui « des collections de l'école de dessin » est rare. Plus généralement, il s'agit de listes fortuites, dressées par exemple au moment du don d'un collectionneur ou du legs d'un professeur, ou établies à l'occasion du déménagement d'une bibliothèque. Il existe aussi des correspondances ${ }^{29}$ qui témoignent de la réalité de la sollicitation et de la réception des œuvres, livres, recueils, etc. ${ }^{30}$. Quand des listes d'acquisitions sont mises au jour, les indications restent évasives. La mention, par exemple, d'un «recueil d'estampes d'après Boucher» ouvre de nombreuses possibilités d'attribution. À Rennes, le "Catalogue des desseins gravés fournis par la province pour l'instruction des élèves de Rennes" rédigé vers 1788 mentionne, entre autres, des académies et des têtes (vieillard, enfant, Christ mort, saint Jérôme) à la fois de Michel-Ange, de Bouchardon et de Charles-Michel-Ange Challe, sans qu'aucune identification ne puisse être suggérée, le fonds originel ayant été dispersé ${ }^{31}$. De la même manière, à Pau, la " Note des modelles aquis par le sieur Dumont relativement à son académie de Dessin le 19 février 1788 " fait état de gravures d'après Sarrazin, de La Fosse, Boucher, Bouchardon, Parrocel et Le Prince avec des indications trop génériques pour en permettre l'identification ${ }^{32}$.

15 Au demeurant, ces listes permettent de dresser un panorama des artistes proposés à la copie des élèves et donc considérés comme exemplaires. Sous l'angle de l'histoire du 
goût, ces références artistiques, même imprécises, désignent les artistes considérés comme utiles à l'apprentissage. Sont-ils uniquement les "grands" noms de maîtres validés par la critique européenne? Des peintres et des sculpteurs français reconnus pour avoir répondu aux commandes royales les plus prestigieuses? Ou bien ceux qui ont laissé une trace de leur production dans la cité ? Quelle place les gloires « locales » tiennent-elles, en comparaison des génies "universels »? Il apparaît qu'à côté des noms habituels émergent des noms inattendus, marquant parfois un attachement quasi affectif à des artistes.

Pour la sculpture, sans surprise, les modèles antiques sont utilisés. Il s'en trouve des copies dans les collections des villes : Torse du Laocoon, Hercule Farnèse et autre Gladiateur Borghèse constituent les modèles les plus répandus. Les copies du Gladiateur Borghèse de Bénigne Gagneraux (1756-1795) pour Dijon ${ }^{33}$ et de Jean-Jacques Bestieu (1754-1842) ${ }^{34}$ pour Montpellier témoignent du long succès des modèles antiques, d'autant plus prégnants que le voyage à Rome est soutenu par les institutions académiques ${ }^{35}$. Les modèles en plâtre ont une conservation fragile et éphémère, comme ce fut le cas pour les moulages offerts à l'académie phocéenne par le célèbre amateur Bergeret de Grancourt (1715-1785). Il rapporte de Rome des moulages du Laocoon et du Gladiateur Borghèse, des têtes, des pieds et de mains jugées "monstrueuses ${ }^{36}$ " qui furent complétés quelques années plus tard par une tête en plâtre d'après l'antique offert par Boyer de Fonscolombe en 1784 et une copie d'un Apollon Farnèse provenant de la collection Borély.

Les grands maîtres des écoles italiennes, flamandes et françaises restent les incontournables des collections des académies d'art. CEuvres inaccessibles pour des académies aux finances modestes, elles entrent dans les collections par le biais des gravures, des copies, qu'elles soient dessinées ou peintes. C'est le cas à Toulouse d'une copie de grande taille, d'après Pierre Paul Rubens, Tomyris reine des Scythes, plongeant la tête de Cyrus dans un vase de sang, attribuée à Nicolas Largillière. Cette œuvre provenant du Cabinet du roi est envoyée à Toulouse en 1768 et vient enrichir une collection de copies comprenant à la fin du siècle aussi bien des Raphaël, Titien ou Véronèse que des Vernet ou Greuze ${ }^{37}$.

Les "hommes illustres" occupent également une place de choix. À cet égard, les collections de bustes en constituent l'expression la plus courante. La liste est longue et croise - particulièrement dans le cas des écoles de dessin insérées dans une académie des sciences, belles-lettres et arts - celle des figures des hommes de sciences et de lettres. Buste de Molière ${ }^{38}$ par Houdon à Montpellier envoyé par l'artiste en 1779, Buste de Rameau (1760) en terre cuite par Jean-Jacques Caffieri à Dijon ${ }^{39}$, Buste de JeanJacques Caffieri par Jean-Baptiste-Joseph Languet de Gergy à Dijon, offert en 1775. Ces "gloires" prennent une coloration particulière dans les villes dont elles sont originaires, d'autant plus lorsqu'il s'agit de peintres ou de sculpteurs. Le cas de Pierre Puget (1620-1694), qui n'a pourtant jamais fait de carrière académique, est à ce titre représentatif. Au sein de l'académie marseillaise, le sculpteur fait l'objet d'un véritable culte qui cherche à positionner l'institution en héritière de son génie créatif. Pourtant, la liste des œuvres de Puget présentes à Marseille reste maigre : un dessin offert par Antoine Gibert, sculpteur de l'arsenal (Marine royale) de Toulon et soucieux d'être attaché à l'académie en 1754 (Musée des Beaux-Arts de Marseille 2016 : 164-165, 173). Aussi l'académie, pour combler ce manque, suscite-t-elle la réalisation de gravures du bas-relief de la Peste de Milan ${ }^{40}$. 

peinture et de sculpture de Paris, les œuvres réalisées par leurs membres les plus éminents constituent un enjeu de taille ${ }^{41}$. Là encore à Marseille, Michel-François Dandré-Bardon compose en 1753 un recueil d'académies d'hommes réalisés par des maîtres de l'académie parisienne pour servir de modèles aux élèves marseillais. Ils s'entraînent dans un premier temps d'après le dessin et non d'après la ronde-bosse ou le modèle vivant. La liste est répartie entre les «anciens" comme Nicolas Bertin, Jouvenet, La Fosse, Houasse, et des artistes contemporains comme Oudry, Natoire, Boucher ou Vanloo dont un certain nombre ont pu être identifiés ${ }^{42}$.

La question du goût est également relayée par une culture livresque dont certains établissements cherchent à être les vecteurs. La présence de bibliothèques, comportant des ouvrages généraux d'histoire, de droit, de sciences naturelles, d'esthétique, des dictionnaires, des traités techniques, des recueils d'antiquités, montre que le projet social des Lumières veut un artiste et un artisan qualifié et éduqué. En 1769, le collectionneur Pierre Adamoli lègue 5600 volumes à l'Académie des sciences, belleslettres et arts de Lyon, composant la partie la plus importante de la bibliothèque de l'établissement ${ }^{43}$. La bibliothèque municipale de Lyon a répertorié plus de 1402 livres provenant de cette collection grâce à la présence de son ex-libris ${ }^{44}$. La présence mêlée dans les cercles académiques de savants et d'érudits, pleinement impliqués dans les programmes de l'école de dessin et dans le développement d'une culture de ville, relève de cet objectif ${ }^{45}$.

\section{Usages et matérialité des œuvres : un défi patrimonial}

La manipulation que suppose le statut de support d'apprentissage explique que nombre des œuvres initialement conservées dans les académies sont perdues ou bien endommagées par les déplacements et les déménagements. On imagine aisément l'ensemble des gestes qui accompagnent une œuvre servant de modèle : la disposer sur un chevalet au milieu de la classe de cours ou sur un pupitre de table, la faire passer d'une personne à l'autre pour observer un détail et le reproduire, montrer voire toucher une peinture pour en révéler la structure et mieux saisir l'épaisseur de la matière picturale, caresser une terre cuite pour comprendre où l'artiste a donné des incises, formé un pli, suggéré une ombre. Les recueils d'estampes, quant à eux, sont souvent démembrés pour que les planches soient distribuées une à une à l'assemblée. Aussi, dès le XVIII ${ }^{\mathrm{e}}$ siècle, les enjeux autour de la conservation émergent et deviennent source de tensions et de crispations. Il est dit notamment que le fonds de dessins de l'école de dessin de Grenoble, apporté par le directeur Jacques-André Treillard et dont la valeur est estimée à 30000 livres, est exagérément "usé et consommé » par les étudiants ${ }^{46}$.

Le cas de la préservation des écorchés est aussi sensible. À Montpellier se trouve un exemplaire saisissant du sculpteur Jean-Antoine Houdon ${ }^{47}$, témoignage d'un modèle présent dans de nombreuses académies et écoles de dessin comme Toulouse ou Grenoble. Sa réputation en Europe est semblable au modèle à succès que produit Edme Bouchardon, dont un exemplaire avait été envoyé à Lille ${ }^{48}$, ou celui de Jean-Pancrace Chastel, visible à Aix ${ }^{49}$. Dans ce cas, la finalité pédagogique de l'œuvre se concentre sur la connaissance du corps humain et non sur la représentation d'une figure historique en mouvement, telle qu'elle s'exprime dans une sculpture traditionnelle en ronde- 
bosse. Par la quantité des copies réalisées, par sa diffusion même et son étrangeté aux côtés des autres statues d'après le vivant, elle devient un modèle d'imitation à succès dans maints établissements européens. Elle forge la réputation de Houdon peut-être plus que ses autres sculptures artistiques.

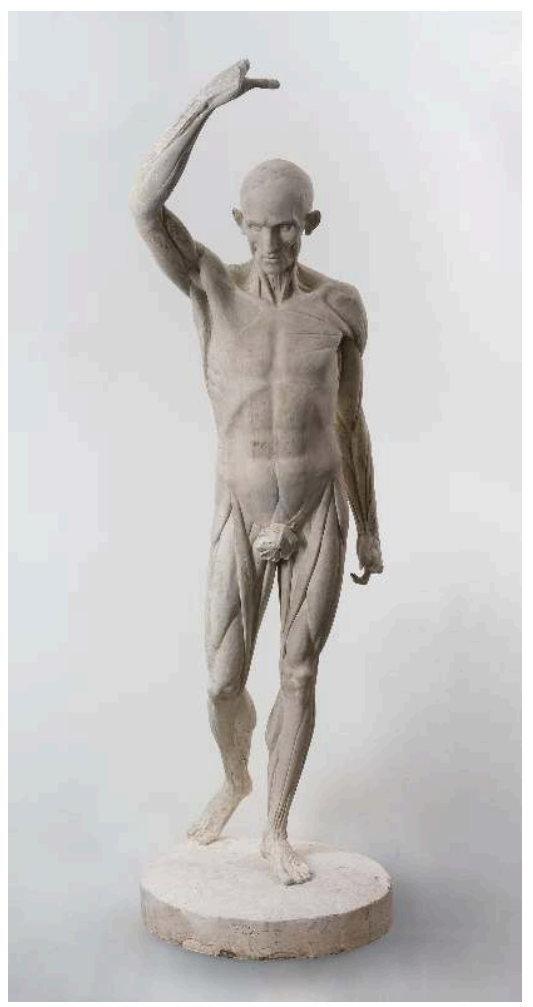

Écorché au bras relevé, Jean-Antoine Houdon, vers 1778, plâtre conservé au musée Fabre (inv. 806.32), Montpellier.

(c) Photo Frédéric Jaulmes, 2017 (Montpellier - Méditerranée Métropole)

En dépit de pertes considérables, une filiation réelle s'organise entre les collections des écoles de dessin et des académies d'art et la naissance des premiers musées des BeauxArts en province. Quand le transfert de la collection n'est pas complet, il arrive qu'une partie soit vendue, ou bien qu'elle suive un parcours naturel de la formation en rejoignant les collections de l'école des beaux-arts du xixe siècle. Les anciens professeurs de l'Ancien Régime conservent leur poste sous la Révolution et l'Empire, au sein de l'école mais aussi des premiers musées des Beaux-Arts. À Aix-en-Provence, par exemple, le peintre marseillais Jean-Antoine Constantin (1756-1844), à la tête de l'institution aixoise depuis 1786, maintient l'enseignement du dessin sous la Révolution puis, après quelques soubresauts, participe en 1806 à la création de la nouvelle école de dessin qui est réunie avec le musée municipal dans l'ancien prieuré de Malte en 1830, l'actuel musée Granet ${ }^{50}$. À Grenoble, la figure de Louis-Joseph Jay (1755-1836) témoigne de la pérennité sous de nouvelles formes des institutions d'Ancien Régime. Membre de la Société des arts de Montpellier, il devient professeur de dessin de l'école centrale de Grenoble et conservateur du nouveau musée. Il suscite achats et legs, incite à faire du premier musée de la ville un lieu d'apprentissage, à l'exemple de l'ancienne école de dessin $^{51}$. À l'occasion d'un voyage en Italie en 1796, il achète 195 dessins et gravures et acquiert les huit premiers tableaux destinés au futur musée des Beaux-Arts grenoblois. À Bordeaux, le peintre Pierre Lacour (1745-1814), recteur de l'académie de peinture, sculpture et architecture, devient premier conservateur du musée des Beaux-Arts. Les 
exemples pourraient être multipliés: le directeur de l'école de dessin de Poitiers François Aujollet-Pagès est le premier conservateur du musée ; à Quimper, le fondateur de l'établissement local, François Valentin, fait office de protecteur des arts pendant la période révolutionnaire. Les premiers jalons posés dans cet article ont montré que si l'essentiel des œuvres repérables aujourd'hui se trouvent dans les musées des Beaux-Arts des villes, il reste d'autres lieux à explorer, en particulier les fonds anciens des bibliothèques municipales et les collections des académies toujours en activité. Dans les écoles des beaux-arts ou de design actuelles, les collections historiques ne sont pas toujours inventoriées. Le cas de Toulouse est symptomatique d'un écartèlement du fonds initial entre le musée des Augustins, le musée Paul-Dupuy et l'Institut supérieur des arts de Toulouse (Isdat).

Ce panorama encore incomplet permet toutefois de dégager de grandes lignes de réflexion. Elles mettent en évidence un aspect de l'histoire des collections nationales jusqu'alors peu valorisé. Alors que l'histoire du patrimoine en France souligne la dissémination organisée des collections venues de Paris dans les nouveaux musées de province, l'histoire des collections des écoles de dessin et académies régionales montre l'origine, voire l'antériorité, d'un processus qui met en avant l'importance des provinces au XVIII ${ }^{\mathrm{e}}$ siècle, avant la centralisation jacobine. Lors de la répartition des saisies révolutionnaires, l'action de l'État s'accorde avec une culture de ville préexistante, celle formée notamment par les écoles de dessin et les académies. L'exemple des tableaux de fleurs de Jean-Baptiste Monnoyer ou du panneau décoratif d'Alexandre-François Desportes, envoyés à Lyon en 1799 résonne par rapport à l'histoire de la Fabrique lyonnaise de soie.

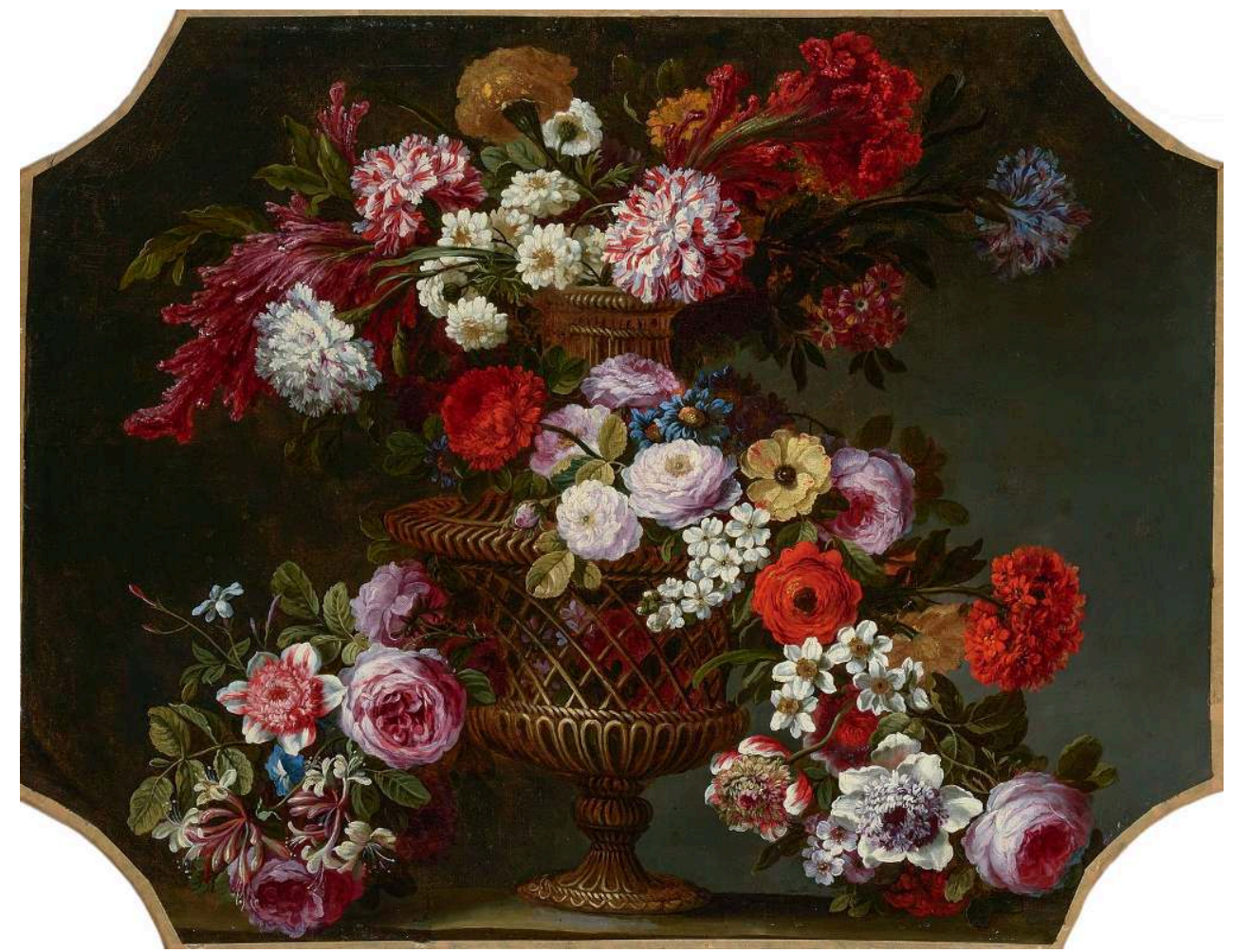

Fleurs dans un vase, peintre anonyme d'après Jean-Baptiste Monnoyer, xvı ${ }^{e}$ siècle, huile sur toile, musée des Beaux-Arts de Lyon. Cette œuvre fait partie d'un lot envoyé par l'État à l'École de dessin de Lyon en 1799.

Reproduction Alain Basset (Lyon MBA). 
Sans verser dans un discours régionaliste qui présenterait ces collections comme l'émanation d'un "génie » local immanent, ces analyses montrent que l'identité des provinces s'est forgée d'apports divers, d'artistes et de collectionneurs, originaires ou au contraire étrangers à leur terre d'implantation. Ils ont marqué par à-coups le paysage citadin. À ce titre, ces collections sont beaucoup plus bigarrées que ne l'ont été les collections de modèles au XIX ${ }^{e}$ siècle, dont la seule et unique vocation était de servir de modèles. Labiles, elles sont représentatives des échanges artistiques, culturels et humains qui s'animent au cœur des cercles académiques au XVIII ${ }^{\mathrm{e}}$ siècle. Elles s'attachent à renouveler leurs modèles et à se tenir informées de l'actualité, en recevant notamment les dernières publications, les revues de savants et les almanachs. Les académies, loin d'être des cénacles fermés qui ne jouent que sur l'entre-soi, sont des ponts dans la société des Lumières.

\section{NOTES}

1. Programme « Les académies d'art et leurs réseaux dans la France pré-industrielle » conduit par Anne Perrin Khelissa et Émilie Roffidal au sein du laboratoire Framespa (UMR CNRS 5136, université Toulouse-Jean-Jaurès. Voir : https://acares.hypotheses.org/ [lien valide en septembre 2020].

2. PERRIN KHELISSA Anne \& ROFFIDAL Émilie, « Mobilité des artistes, dynamique des institutions : dessiner la cartographie des échanges. Compte rendu des journées d'étude des 9-10 novembre 2017 », Les Papiers d'ACA-RES, « Actes des journées d'étude 2 », actes des journées d'étude à la Maison de la recherche UT2J (Toulouse, 9-10 novembre 2017), 2018 [en ligne], https://acares.hypotheses.org/actes-des-journees-detude-2 [lien valide en septembre 2020].

3. https://www.pop.culture.gouv.fr/notice/joconde/03110000272

4. POULOT Dominique, Musée, nation, patrimoine. 1789-1815, Paris, Gallimard, coll. « Bibliothèque des histoires ", 1997, p. 85-89. POULOT Dominique, Patrimoine et musées. L'institution de la culture, Paris, Hachette, coll. « Carré histoire » 2014 [2001], p. 46.

5. LAHALLE Agnès, Les Écoles de dessin au XVIII ${ }^{e}$ siècle. Entre arts libéraux et arts mécaniques, Rennes, Presses universitaires de Rennes, coll. « Histoire », 2006. Pour un état de l'art sur le sujet, voir la rubrique «État du champ scientifique » sur la page Hypothèses du programme ACA-RES (https:// acares.hypotheses.org/etat-du-champ-scientifique), ainsi que la bibliographie sous Zotero mise à disposition (https://acares.hypotheses.org/bibliotheque-numerique).

6. Exposition virtuelle réalisée grâce au soutien de l'université Toulouse - Jean-Jaurès et de l'INHA par une Carte blanche 2019 auquel furent associés de jeunes chercheurs en histoire de l'art (https://acares.hypotheses.org/le-projet). À ce titre, nous remercions Loïc Cabrita, Émilie Ginestet, Manon Migot, Mathilde Roy, Caroline Ruiz, Nelly Vi-Tong et surtout Tara Cruzol pour leur contribution. Voir l'exposition en ligne : http://acares.univ-tlse2.fr/ [lien valide en janvier 2021].

7. Mentionnons notamment pour les premiers : MUSÉE DES BEAUX-ARTS DE VALENCIENNES, L'Académie de peinture et de sculpture à Valenciennes au XVIII 'siècle, catalogue d'exposition au musée des Beaux-Arts de Valenciennes, Valenciennes, Société des amis du musée de Valenciennes, 
1986 ; MUSÉE DES BEAUX-ARTS TOURS, Dessins, $X V^{e}-X X^{e}$ siècle. La collection du musée de Tours, catalogue d'exposition au musée des Beaux-Arts de Tours, Tours, musée des Beaux-Arts, 2001 ; BERGOT François, Trésors de l'Académie des sciences, belles-lettres et arts de Rouen, Rouen / Bonsecours, Académie des sciences, belles-lettres et arts de Rouen / éditions Point de vues, 2009. Et pour les seconds : GOBET Aude, «Une sociabilité du dessin au XVIII ${ }^{\mathrm{e}}$ siècle : artistes et académiciens à Rouen au temps de Jean-Baptiste Descamps (1715-1791) », thèse d'histoire de l'art soutenue à l'université Paris-1 - Panthéon-Sorbonne (Paris), 2007 ; QUIQUEMPOIS Olivier, « La collection de l'Académie royale de peinture, sculpture et architecture de Toulouse ", mémoire de recherche de $2^{\mathrm{e}}$ année de $2^{\mathrm{e}}$ cycle en histoire de l'art appliquée aux collections, École du Louvre (Paris), 2012 ; GUILLIN Marjorie, « "L'anéantissement des arts en province” ? L'académie royale de peinture, sculpture et architecture de Toulouse au XVIII ${ }^{\mathrm{e}}$ siècle (1751-1793) ", thèse d'histoire de l'art sous la direction de Pascal Julien soutenue à l'université Toulouse - Jean-Jaurès (Toulouse), 2013. Voir enfin le travail de doctorat récemment soutenu par Nelly Vi-Tong.

8. Voir MUSÉE DES BEAUX-ARTS DE MARSEILLE, 2016, Marseille au XVIII ${ }^{e}$ siècle. Les années de l'Académie de peinture et de sculpture, 1753-1793, catalogue de l'exposition au musée des Beaux-Arts (Marseille, 17 juin-16 octobre 2016), Paris / Marseille, Somogy / Musées de Marseille, 2016. Voir également HILAIRE Michel \& STÉPANOFF Pierre (dir.), Le Musée avant le musée. La Société des beauxarts de Montpellier (1779-1787), catalogue de l'exposition au musée Fabre (Montpellier, 9 décembre 2017-11 mars 2018), Gand / Montpellier, Snoeck / Musée Fabre, 2018. Des programmes de recherche récents se sont également consacrés à ce thème : notamment « Académie / Académies » du Centre Georges-Chevrier sur le fonds de l'école de dessin de Dijon (base de données accessible par le lien : http://tristan.u-bourgogne.fr/Academie/index.html), et la base Agorha consacrée par l'Institut national d'histoire de l'art (INHA) aux envois de Rome (https:// agorha.inha.fr/inhaprod/ark:/54721/00180).

9. FONTAINE André, Les Collections de l'Académie royale de peinture et de sculpture, Paris, H. Laurens, 1910. BRUGEROLLES Emmanuelle, L'Académie mise à nu. L'école du modèle à l'Académie royale de peinture et de sculpture, catalogue de l'exposition à l'École nationale supérieure des beaux-arts (Paris, 26 octobre 2009-29 janvier 2010), Paris, Éditions des Beaux-Arts de Paris, coll. «Carnets d'études / École nationale supérieure des beaux-arts, Cabinet des dessins Jean-Bonna / Collection Mathias-Polakovits », 2009.

10. Le projet d'école date de 1778.

11. SNYERS Alain, 1782-2004. L'École de dessin, de Maurice-Quentin de La Tour à aujourd'hui, SaintQuentin, Ville de Saint-Quentin, 2004 [disponible en ligne via la bibliothèque numérique ACARES], https://acares.hypotheses.org/bibliotheque-numerique [lien valide en septembre 2020].

12. HOUDOY Jules, «L'Académie des arts de Lille », Mémoires de la Société des sciences de Lille, $4^{\text {e }}$ série, t. 3, 1877, p. 281-306. DUTHOY Jean-Jacques \& OURSEL Hervé, « L'enseignement à l'Académie des arts de Lille au XVIII ${ }^{\mathrm{e}}$ siècle (à propos d'un album de dessins de la bibliothèque municipale de Lille) », Revue du Nord, t. 71, n² 281, 1989, p. 377-399 [disponible en ligne], https:// www.persee.fr/doc/rnord_0035-2624_1989_num_71_281_4452 [lien valide en septembre 2020]. OURSEL Hervé, « L'Académie des Arts de Lille au XVIII ${ }^{\mathrm{e}}$ siècle », in BOSCHLOO Anton W. A. et al., Academies of Art. Between Renaissance and Romanticism, La Haye, SDU Uitgeverij, coll. « Leids kunsthistorisch jaarboek », 1989, p. 244-253.

13. SNYERS Alain, 1782-2004. L'École de dessin..., p. 5.

14. DUTHOY Jean-Jacques \& OURSEL Hervé, «L'enseignement à l'Académie des arts de Lille au XVIII ${ }^{\mathrm{e}}$ siècle... ", 1989.

15. Les dessins, mentionnés dans un inventaire de l'an II (1793-1794), sont ensuite dispersés. Au milieu du XIX ${ }^{\mathrm{e}}$ siècle, le musée des Beaux-Arts de Marseille rachète certains dessins, d'autres sont 
conservés au Louvre ou dans des collections particulières. Voir MUSÉE DES BEAUX-ARTS DE MARSEILLE, Marseille au XVIII ${ }^{e}$ siècle, 2016, p. 139, note 92.

16. Voir, entre autres, cette vocation clairement affichée à l'école de dessin d'Angers : JAMESSARAZIN Ariane, «Les écoles de dessin à Angers », Les Papiers d'ACA-RES, « Brefs historiques », 2017 [en ligne], https://acares.hypotheses.org/les-papiers-daca-res/bref-historique [lien valide en septembre 2020].

17. MASSOUNIE Dominique, «La place de l'architecture et de l'École des arts de Jacques-François Blondel dans l'histoire des académies artistiques provinciales du XVIII ${ }^{\mathrm{e}}$ siècle ", Les Papiers d'ACA$R E S$, « Actes des journées d'étude 3 », actes des journées d'étude à l'Hôtel des sociétés savantes (Rouen, 29-30 novembre 2018), 2019 [en ligne], https://acares.hypotheses.org/actes-desjournees-detude-3 [lien valide en septembre 2020].

18. TAILLARD Christian, «L'Académie de peinture de Bordeaux (1768-1792) », in Musée des Beaux-Arts de Bordeaux, Le Port des lumières, vol. 1, La peinture à Bordeaux (1750-1800), catalogue de l'exposition au musée des Beaux-Arts (Bordeaux, 1989), Bordeaux, William Blake \& Co., 1989, p. 13-22. BERDU Lucas, «L'École de dessin et l'Académie de peinture, sculpture et architecture civile et navale de Bordeaux », Les Papiers d'ACA-RES, « Brefs historiques », [en ligne], https:// acares.hypotheses.org/les-papiers-daca-res/bref-historique [lien valide en septembre 2020].

19. GOBET Aude, « Une sociabilité du dessin au XVIII siècle... », 2007. MORVAN BECKER Frédéric, «L'École gratuite de dessin de Rouen, ou la formation des techniciens au XVIII ${ }^{\mathrm{e}}$ siècle ", thèse de doctorat d'histoire sous la direction de Philippe Minard soutenue à l'université Paris-8 - SaintDenis (Paris), 2010 [disponible en ligne], https://octaviana.fr/document/161049583\#? $\mathrm{c}=0 \& \mathrm{~m}=0 \& \mathrm{~s}=0 \& \mathrm{cv}=0$ [lien valide en septembre 2020].

20. Voir https://www.pop.culture.gouv.fr/notice/joconde/05620001640

21. S. N., Catalogue des ouvrages exposés au Salon de l'hôtel de ville par l'Académie royale de peinture, sculpture et architecture, le 18 du mois de mai 1773, et pendant les huit jours suivants, Toulouse, Le Capitole, 1773, $\mathrm{n}^{\circ}$ 114. MESURET Robert (dir.), Les Expositions de l'Académie royale de Toulouse de 1751 à 1791, Toulouse, Espic, 1972, p. 233. FIOZZI David, Les Tableaux hollandais des XVII et XVIII siècles du musée des Augustins, catalogue d'exposition (Toulouse, 18 décembre 2004-9 mai 2005) et catalogue raisonné, Toulouse, musée des Augustins, 2004, p. 93, n² 29. QUIQUEMPOIS Olivier, « La collection de l'Académie royale de peinture, sculpture et architecture de Toulouse », 2012, p. 108-109.

22. LACOUR Pierre, Notice des tableaux et figures exposés au musée de la Ville de Bordeaux, Bordeaux, s. n., 1821, p. $67, \mathrm{n}^{\circ}$ 72. Ce tableau est depuis 1801 dans les collections du musée des Beaux-Arts de Bordeaux (Bx E 375).

23. MAËS Gaëtane, « Le Salon de Paris : un modèle pour la France et pour les Français au $\mathrm{XVIII}^{\mathrm{e}}$ siècle ? ", in PICHET Isabelle (dir.), Le Salon de l'Académie royale de peinture et sculpture. Archéologie d'une institution, Paris, Hermann, coll. « République des lettres. Symposiums », 2014, p. 33-56.

24. Voir l'article de Nelly Vi-Tong accompagné de la bibliographie antérieure se rapportant à cette école : VI-TONG Nelly, «L'École de dessin de Dijon », Les Papiers d'ACA-RES, « Brefs historiques », 2017 [en ligne] https://acares.hypotheses.org/les-papiers-daca-res/bref-historique [lien valide en septembre 2020].

25. HILAIRE Michel \& STÉPANOFF Pierre (dir.), Le Musée avant le musée, 2018, p. 86-87 ; JOUBIN André, Catalogue des peintures et des sculptures exposées dans les galeries du musée Fabre de la ville de Montpellier, Paris, Imprimerie Blondel la Rougery, 1926, n 783 ; LERIBAULT Christophe, JeanFrançois de Troy, 1679-1752, Paris, Arthena, 2002, p. 125 ; ZEDER Olivier, Peintures françaises du musée 
Fabre. Catalogue raisonné, vol. 1, De la Renaissance à la Régence, Paris / Montpellier, Somogy / Musée Fabre, 2011 : 208, n 128.

26. HÉNAULT Maurice, «L'Académie de peinture et de sculpture de Valenciennes ses origines (1785-1793) ", Réunion des sociétés savantes des départements, 1914, p. 222. CHAMPION Jean-Loup (dir.), Mille sculptures des musées de France, Paris, Gallimard, 1998, p. 230.

27. HÉNAULT Maurice, «L'Académie de peinture et de sculpture de Valenciennes... », 1914.

28. Voir la notice sur Jérôme Preudhomme et ses tableaux à Saint-Quentin : https:// www.pop.culture.gouv.fr/notice/joconde/07930000871 [lien valide en septembre 2020]. Voir également S. N., Catalogue de la collection des pastels de Delatour et des autres tableaux de différens maîtres se trouvant dans les salles d'étude de l'école gratuite de dessin de Saint-Quentin, Saint-Quentin, Imprimerie de [Louis] Adolphe Moureau, 1856, p. 12. Voir enfin MERSON Olivier, « Revue européenne. Musée de Saint-Quentin », Journal de Saint-Quentin, 1861 (15 mars).

29. Les lettres numérisées sont accessibles sur le site ACA-RES: https://acaresarchives.nakalona.fr/ [lien valide en septembre 2020].

30. ROFFIDAL Émilie, «Correspondance romaine d'une académie de province : l'Académie de peinture et de sculpture de Marseille », in ROLFI OZVALD Serenella \& MAZZARELLI Carla (dir.), Il Carteggio d'artista. Fonti, questioni, ricerche tra XVII $-\mathrm{XIX}^{e}$ secolo, Cinisello Balsamo, Silvana Editoriale, 2019, p. 188-199 [disponible en ligne via la bibliothèque numérique ACA-RES], https:// acares.hypotheses.org/bibliotheque-numerique [lien valide en septembre 2020].

31. AD Ille-et-Vilaine (C 4919, « [Inventaires de dessins, gravures, modèles en plâtre pour l'École de dessin de Rennes, 1786-1788]») [disponible en ligne], https://acares-archives.nakalona.fr/ items/show/577 [lien valide en septembre 2020].

32. AD Pyrénées-Atlantiques (C 1341, « [Liste des modèles acquis pour l'école de dessin de Pau, 19 février 1788] »). Document transcrit par Florie Valton, collationné et disponible en ligne : https://acares-archives.nakalona.fr/items/show/2039 [lien valide en septembre 2020].

33. Musée des Beaux-Arts de Dijon (inv. ES 45).

34. Musée Atger, université de Montpellier (inv. MA 923). Voir CLAPARÈDE Jean, « Houdon et la Société des beaux-arts de Montpellier (1779-1784) », Études héraultaises, nouvelle série, n 9, 1993, p. 106-107 [disponible en ligne], https://www.etudesheraultaises.fr/publi/houdon-et-la-societedes-beaux-arts-de-montpellier-1779-1784_j-claparede/ [lien valide en septembre 2020].

35. La question du voyage romain dans le cadre académique a été notamment traité lors des $2^{\text {es }}$ journées d'étude du programme ACA-RES : « Mobilité des artistes, dynamique des institutions : dessiner la cartographie des échanges ", organisées à la Maison de la recherche UT2J (Toulouse, 9-10 novembre 2017 (https://acares.hypotheses.org/actes-des-journees-detude-2)

36. Marseille, BMVR [bibliothèque municipale à vocation régionale], Archives de l'académie de peinture et de sculpture de Marseille (Ms 988, t. 10, f. 128-129, « [Lettre de Dandré-Bardon à l'académie de peinture de Marseille, Paris, 10 juin 1781] ») [document collationné et disponible en ligne], https://acares-archives.nakalona.fr/items/show/1391 [lien valide en septembre 2020]. Voir également ROFFIDAL Émilie, « L'École de dessin d'Aix-en-Provence », Les Papiers d'ACA-RES, «Brefs historiques », 2017, note 27 [en ligne], https://acares.hypotheses.org/files/2017/03/ roffidal-2017.pdf [lien valide en septembre 2020] ; MUSÉE DES BEAUX-ARTS DE MARSEILLE, Marseille au XVIII siècle, 2016, p. 118).

37. QUIQUEMPOIS Olivier, « La collection de l'Académie royale de peinture, sculpture et architecture de Toulouse », 2012, p. 19, 146-147, 150-151.

38. BAJOU Valérie, « Houdon in the Musée Fabre », Apollo, n 129, 1989, p. 23-26, ill. 3. 
39. Cette terre cuite exécutée en 1760 par Jean-Jacques Caffieri, sculpteur d'origine italienne, est une œuvre préparatoire à un marbre exposé au Salon de 1761. Voir le magazine Dossier de l'art, $n^{\circ}$ 269, « Réouverture du musée des Beaux-Arts de Dijon », mai 2019, p. 46.

40. Sur l'œuvre, consulter la notice: GEORGET Luc, « Catalogue », in VIALE Marie-Paule (dir.), Pierre Puget. Peintre, sculpture, architecte, 1620-1694, catalogue de l'exposition organisée au musée des Beaux-Arts / Centre de la Vieille-Charité (Marseille, 28 octobre 1994-30 janvier 1995), Marseille / Paris, Musées de Marseille / RMN, 1994, p. 144-147.

41. Sur ces liens Paris-provinces, voir PERRIN KHELISSA Anne \& ROFFIDAL Émilie, « Fonder les institutions artistiques : l'individu, la communauté et leurs réseaux en question ", Les Papiers d'ACA-RES, « Actes des journées d'étude 1 », actes des journées d'étude au Centre allemand d'histoire de l'art (Paris, 8-9 décembre 2016), 2017 [en ligne], https://acares.hypotheses.org/lespapiers-daca-res/actes-des-journees-detude [lien valide en septembre 2020]. Voir également PERRIN KHELISSA Anne \& ROFFIDAL Émilie, « Réseaux des académies d'art provinciales et dynamiques des circulations au XVIII ${ }^{\mathrm{e}}$ siècle ", actes du congrès annuel du Centre allemand d'histoire de l'art (Paris, 2018), à paraître.

42. BÉDARD Sylvain, « Un envoi de dessins de l'Académie royale de peinture et de sculpture à Marseille en 1754 », Bulletin de la Société de l'histoire de l'art français, « Année 2003 », 2004, p. 209-219. BÉDARD Sylvain, « Modèles parisiens. Un lot de figures académiques pour Marseille », in MUSÉE DES BEAUX-ARTS DE MARSEILLE, Marseille au XVIII ${ }^{e}$ siècle, 2016, p. 159-185.

43. SORDET Yann, Pierre Adamoli et ses collections. L'amour des livres au siècle des Lumières, Paris, École des chartes, coll. « Mémoires et documents de l'École des chartes », 2001.

44. https://catalogue.bm-lyon.fr

45. PERRIN KHELISSA Anne, « Le traité de peinture de Donat Nonnotte, ancien élève de François Le Moyne. Discours prononcé à l'académie de Lyon entre 1754 et 1779 ", Mémoires de l'Académie des sciences, belles-lettres et arts de Lyon, $4^{\text {e }}$ série, t. 10, 2011, p. 221-371 [disponible en ligne via la bibliothèque numérique ACA-RES], https://acares.hypotheses.org/bibliotheque-numerique [lien valide en septembre 2020].

46. Archives départementales de l'Isère (D 3). Voir BENHAMOU Reed, « Art et utilité. Les écoles de dessin de Grenoble et de Poitiers ", Dix-huitième siècle, n²3, " Physiologie et médecine ", 1991, p. 428 [disponible en ligne], https://www.persee.fr/doc/dhs_0070-6760_1991_num_23_1_1828 [lien valide en septembre 2020]. Voir également HUMBERT Candice, « L'École de dessin de Grenoble », Les Papiers d'ACA-RES, « Brefs historiques », 2017a [en ligne], https:// acares.hypotheses.org/files/2017/03/humbert-2017.pdf [lien valide en septembre 2020].

47. Cet Écorché se trouve aujourd'hui dans les collections du musée Fabre (inv. 806.32). Voir CLAPARÈDE Jean, « Houdon et la Société des beaux-arts de Montpellier (1779-1784) », 1993, p. 40 ; HILAIRE Michel \& STÉPANOFF Pierre (dir.), Le Musée avant le musée, 2018: 102 ; CÉSAR Flore, « Des arts dans une ville de sciences, des sciences dans une école d'art : la Société des beaux-arts de Montpellier, 1777-1784 ", Les Papiers d'ACA-RES, « Actes des journées d'étude 3 », actes des journées d'étude à l'Hôtel des sociétés savantes (Rouen, 29-30 novembre 2018), 2019 [en ligne], https://acares.hypotheses.org/actes-des-journees-detude-3 [lien valide en septembre 2020].

48. LAHALLE Agnès, Les Écoles de dessin au XVIII siècle. 2006, p. 229.

49. Aix-en-Provence, musée Granet (inv. S-853.5.1). Voir à ce sujet CHASTEL Jean-Pancrace \& BOUCHE Charles-François, Mémoire présenté par le sieur Chastel, sculpteur de la ville d'Aix, à Nosseigneurs et Messieurs des États de Provence convoqués à Lambesc le 4 décembre 1774, Aix-enProvence, Esprit David, 1774, p. 13, note g. (mémoire conservé à la bibliothèque Méjanes d'Aixen-Provence, Anc. / $8^{\circ}$ pcs 10712). Voir également MARAL Alexandre (dir.), Sculptures, 2003, 
p. 64-65 ; et TAUZIÈDE-ESPARIAT Maël, « Des concurrentes de l'Académie de peinture et de sculpture de Marseille ? Les écoles de dessin et de sculpture d'Aix d'après un Mémoire du sculpteur Jean-Pancrace Chastel (1774) », Rives méditerranéennes, n56, « L'Académie de peinture, sculpture et architecture civile et navale de Marseille $17531793 », 2018$, p. 141 [disponible en ligne], https://journals.openedition.org/rives/5416 [lien valide en septembre 2020].

50. Pour une présentation synthétique de cette école, voir ROFFIDAL Émilie, « L'École de dessin d'Aix-en-Provence », 2017. Pour plus de détails, voir COSTE Numa, « Les origines de l'École de dessin et du musée d'Aix-en-Provence ", Réunion des sociétés des beaux-arts des départements, 1905, p. 268-276 ; COSTE Numa, « Les origines de l'École de dessin et du musée d'Aix-en-Provence », Réunion des sociétés des beaux-arts des départements, 1906, p. 289 ; MARAL Alexandre (dir.), Sculptures. La galerie du musée Granet, Aix-en-Provence / Paris, musée Granet / Somogy, 2003, p. 10-25.

51. CLERC Marianne, «Peindre à Grenoble au XVIII ${ }^{\mathrm{e}}$ siècle : motivations et désillusions d'un "talent qui n'est point éminent" ", in LETHUILLIER Jean-Pierre (dir.), La Peinture en province. De la fin du Moyen Âge au début du XX $X^{e}$ siècle, actes du colloque de l'université Rennes-2, Rennes, Presses universitaires de Rennes, 2002, p. 107-113. HUMBERT Candice, « L'École de dessin de Grenoble », 2017a. HUMBERT Candice, « Louis-Joseph Jay (Saint-Hilaire-de-la-Côte [Isère] 1755-Vienne [Isère] 1836) : de Montpellier à Grenoble, quel parcours pour quelles ambitions ? », Les Papiers d'ACA-RES, « Actes des journées d'étude 2 », 2017b [en ligne], https://acares.hypotheses.org/actes-desjournees-detude-2 [lien valide en septembre 2020].

\section{RÉSUMÉS}

Dans l'histoire des collections françaises, celles issues des écoles de dessin et des académies d'art $\mathrm{du} \mathrm{XVIII}^{\mathrm{e}}$ siècle ont un statut à part. Leur spécificité tient tout d'abord à leur mode de constitution. Elles résultent d'actions collectives mettant en réseau des individus aux statuts et aux intérêts divers: artistes, amateurs et acteurs de la vie économique et politique. Elles traduisent une certaine identité artistique, qui lie formation et création dans le domaine des beaux-arts et des arts manufacturés. La synthèse ici présentée s'inscrit dans les recherches du programme ACARES (Les académies d'art et leurs réseaux dans la France préindustrielle).

Collections from 18th century drawing schools and art academies have a unique status in the history of French collections. Their specificity relies mainly on the methods used to create them. They are the results of collective actions that created a network of individuals of varying statuses and interest: artists, art lovers, economical and political actors. They show a specific artistic identity, which links collection development to creation in the fine arts and manufactured arts domains. The synthesis written here is a part of the researches by the ACA-RES Program ("Academies of fine art and their relationships in pre-industrial France"). 
INDEX

Mots-clés : collections, académies, écoles de dessin, xviiie siècle, pédagogie, modèles artistiques, matérialité des œuvres

Keywords : collections, academies, drawing schools, 18th century, pedagogy, artistic models, materiality of works

\section{AUTEURS}

\section{ANNE PERRIN KHELISSA}

Maître de conférence, membre du Laboratoire Framespa (UMR CNRS 5136, université Toulouse Jean-Jaurès)

anne.perrin-khelissa@univ-tlse2.fr

\section{ÉMILIE ROFFIDAL}

Chargée de recherche CNRS, laboratoire Framespa (UMR CNRS 5136, université Toulouse-JeanJaurès)

emilie.roffidal@univ-tlse2.fr 